\section{ORIGINAL RESEARCH}

\author{
M.H. Sombekke \\ M.M. Vellinga \\ B.M.J. Uitdehaag \\ F. Barkhof \\ C.H. Polman \\ D. Arteta
}

D. Tejedor

A. Martinez

J.B.A. Crusius

A.S. Peña

J.J.G. Geurts

H. Vrenken

\title{
Genetic Correlations of Brain Lesion Distribution in Multiple Sclerosis: An Exploratory Study
}

BACKGROUND AND PURPOSE: In MS, the total brain lesion volume and spatial distribution of lesions across the brain vary widely among individual patients. We hypothesized that spatial distribution may be partially driven by genetic predisposition, and we aimed to explore relations among candidate genes and the spatial distribution of white matter brain lesions in MS

MATERIAL AND METHODS: Genotypes of 69 SNPs in 208 patients with MS were related to the spatial distribution of T2 brain lesions. Lesions were manually outlined on MR images, and binary lesion masks were produced and registered to a common space. With Randomise software, the lesion masks were related to genotype by using a voxelwise nonparametric GLM approach, followed by clusterwise analysis. We used a DNA chip with SNPs selected from the literature on MS susceptibility, severity, and phenotypes.

RESULTS: For 11 of these SNPs, 1 of the genotypes expressed significant clusters of increased or decreased lesion probability in varying, predominantly periventricular, brain regions. When we statistically controlled the voxelwise analyses for effects of total brain lesion volume, only 1 SNP remained significant: rs2227139, located within the MHC class II region. This SNP retained its periventricular cluster of significantly increased lesion probability for the heterozygote genotype.

CONCLUSIONS: Heterozygosity of rs2227139 (MHC class II region) is associated with increased right frontal periventricular lesion probability $(P<.01)$. Ten other SNPs showed associations between genotype and spatial lesion distribution that are partly explained by total lesion volume.

\begin{abstract}
ABBREVIATIONS: BTNL2 = butyrophilin-like 2; CCL5 = chemokine (C-C motif) ligand 5; CCR5 = chemokine (C-C motif) receptor 5 ; $C N S=$ central nervous system; $C R Y A B=\alpha$ B crystallin; $E A E=$ experimental autoimmune encephalomyelitis; EDSS = Expanded Disability Status Scale; $F A S=$ tumor necrosis factor receptor superfamily, member 6; FLIRT = FMRIB Nonlinear Image Registration Tool; $\mathrm{FSL}=$ FMRIB Software Library; FMRIB = Functional MR Imaging of the Brain; GLM = general linear model; HLA = human leucocyte antigen; HLA-DRB1 = major histocompatibility complex, class II, DRB1 (Homo sapiens); IFNGR2 = interferon gamma receptor 2 (interferon $\gamma$ transducer 1); IQR = interquartile range; $\mathrm{LPM}=$ lesion probability mapping; $\mathrm{MAF}=$ minor allele frequency; $\mathrm{MHC}=$ major histocompatibility complex; $\mathrm{MS}=$ multiple sclerosis; $\mathrm{NADH}=$ nicotinamide adenine dinucleotide; NDUFS7 = NADH dehydrogenase (ubiquinone) Fe-S protein 7; PNMT = phenylethanolamine $N$-methyltransferase; PPMS = primary-progressive MS; RRMS = relapsing-remitting MS; rs-nr = RefSNP accession ID; $\mathrm{SNP}=$ single nucleotide polymorphism; SPMS = secondary-progressive MS; UCP2 = uncoupling protein 2
\end{abstract}

M $S$ is a multifocal inflammatory demyelinating disease of the CNS. MS lesions can be found throughout the entire CNS parenchyma, histopathologically characterized by inflammation, demyelination, and axonal loss resulting in a highly variable clinical presentation. Lucchinetti et $\mathrm{al}^{1}$ showed the histopathologic heterogeneity of MS lesions among patients with MS, with a distinct homogeneity within each pa-

Received June 6, 2010; accepted after revision September 2.

From the Departments of Neurology (M.H.S., M.M.V., B.M.J.U., C.H.P.), Epidemiology and Biostatistics (B.M.J.U.), Radiology (F.B., J.J.G.G., H.V.), Pathology, Laboratory of Immunogenetics (J.B.A.C., A.S.P.), Pathology (J.J.G.G.), and Physics and Medical Technology (H.V.), VU University Medical Center, Amsterdam, the Netherlands; and Progenika Biopharma, S.A. (D.A., D.T., A.M.), Derio, Spain.

Both M.H. Sombekke and M.M. Vellinga contributed equally to this work.

This work was supported by the Dutch MS Research Foundation through a pilot project grant (grant 05-566). The MS Center Amsterdam is supported by the Dutch MS Research Foundation through a program grant (grants 05-358c and 02-358b). The study was further supported by a corporate sponsor (Progenika Biopharma S.A.), which performed DNA analyses. Design and conduct study, including selection of SNPs for DNA chip, were supported by academics and corporate sponsor jointly. The DNA chip was intellectually protected by the corporate sponsor. Statistical analyses and collection management analysis and interpretation of the data were performed by academics. Preparation, review, and approval of the first draft of the manuscript were performed by academics; the corporate sponsor reviewed and approved the current manuscript. tient, which might partially depend on the immunogenetic background of the individual patient with MS. ${ }^{1,2}$

The spatial distribution of MS lesions across the brain is not homogeneous, showing particularly high frequencies in the periventricular white matter. Moreover, this spatial distribution is highly variable among patients. ${ }^{3-5}$ LPM studies in combination with a nonparametric GLM approach have proved useful in studying associations between lesion distribution and clinical disability parameters. This voxelwise method does not require a priori assumptions. Previous studies have shown differences in lesion distribution across disease subtypes and disability status, ${ }^{5-7}$ as well as among different lesion subtypes: T1 gadolinium-enhancing lesions showed a

Paper previously presented as a poster at: Annual Meeting of the European Committee for Treatment and Research in Multiple Sclerosis, September 9-12, 2009; Dusseldorf, Germany.

Please address correspondence to Madeleine H. Sombekke, MD, VU University Medical Center, Department of Neurology, De Boelelaan 1117, 1081 HV Amsterdam, the Netherlands; e-mail: m.sombekke@vumc.nl

三E Indicates article with supplemental on-line table.

DOI 10.3174/ajnr.A2352 
spatial distribution differing from nonenhancing T2 lesions and from $\mathrm{T} 1$ hypointense lesions (black holes). ${ }^{8,9}$

We hypothesized that spatial lesion distribution might depend on individual immunogenetic background. Although genotype has been shown to influence MS susceptibility in several studies, ${ }^{10-12}$ there are relatively few studies of genotype-phenotype associations in MS, and positive results await confirmation in larger studies. ${ }^{13-18}$

We suspect a genetic influence on the anatomic distribution of MS lesions for 3 main reasons: First, studies confirm a tendency of patients to develop relapses in the same locations. ${ }^{19,20}$ Moreover, in relatives who have MS, lesions have been observed more frequently in similar locations. ${ }^{14}$ This tendency for localized exacerbations could be genetically predetermined. Second, in EAE (the animal model used to study MS), involvement of a genetic factor was already demonstrated in lesion distribution. Butterfield et $\mathrm{al}^{21}$ showed that in mice EAE, the lesions in the spinal cord and brain were controlled by different quantitative trait loci. Third, recently we found that carriership of $H L A-D R B 1^{*} 1501$ is associated with more lesions within the spinal cord, but not with the total lesion volume in the brain. ${ }^{22}$ This allele, also known to be involved in MS susceptibility, is hence suggested to specifically affect lesion development in the spinal cord, a particular region of the central nervous system. Similarly, lesion development in certain regions inside the brain may be partially dependent on genetic parameters. A recent study found that the $H L A-D R B 1^{\star} 1501$ allele did not have an effect on lesion distribution. ${ }^{23}$ We hypothesized that an effect on lesion distribution may be even more likely for genes associated with disease phenotype.

To explore this hypothesis, the present study compared spatial lesion distribution on MR imaging between different genotypes of a set of genes selected on the basis of their potential contribution to phenotypes of MS in a large cohort of patients with MS, by using LPM.

\section{Materials and Methods}

\section{Patients}

This cross-sectional study used clinical and genetic data and MR images of 208 white patients sampled from MS natural history studies at our center. Patients were included on the basis of availability of brain MR imaging and DNA material. The selected patients are a subgroup of those in whom the clinical correlates of brain lesion distribution were studied previously. ${ }^{7}$ All had a diagnosis of MS according to the Poser criteria, ${ }^{24}$ McDonald criteria, ${ }^{25}$ or revised McDonald criteria, ${ }^{26}$ depending on the date of data acquisition. EDSS $^{27}$ scores were obtained within a median interval of 0.0 (IQR, 0.0-4.8) months from MR imaging. Approval by the local ethics review board was obtained, and all participants gave informed consent.

\section{Selection of SNPs}

PubMed (http://www.ncbi.nlm.nih.gov/sites/entrez) and Genetic Association (http://geneticassociationdb.nih.gov) data bases were searched for genes and polymorphisms with suggested involvement in pathogenesis, prognosis, and treatment response in MS. Resulting polymorphisms were confirmed and associated with an identifier by using the dbSNP data base (www.ncbi.nlm.nih.gov/SNP). Their frequency in the white population was assessed by using the HapMap project (http://www.hapmap.org/index.html.en). Nucleotide sequences for the design of allele-specific probes and polymerase chain reaction primers were retrieved in the SNPper data base (http://snpper. chip.org/bio). Sequence-specific probes and primers were designed by using Primer3 software (available free at http://frodo.wi.mit.edu). If a polymorphism was not present in the data base, position and sequences were established by performing a blast search (http://blast.ncbi.nlm.nih. gov/Blast.cgi) by using data available in the literature. SNPs within the MHC class II region were predominantly selected on the basis of the described linkage disequilibrium with the $H L A-D R B 1^{*} 1501$ allele. ${ }^{28,29}$ All genetic data bases were accessed in 2006. The SNP rs numbers and gene symbols were actualized on February 9, 2009.

A total of 80 validated polymorphisms located in 44 different genes were finally chosen (On-line Table). We excluded 11 of these 80 SNPs: 5 because they were monomorphic and 6 because they had a minor allele frequency below 5\%. Deviations from Hardy-Weinberg equilibrium were determined by $\chi^{2}$ testing.

\section{Genotyping}

Genomic DNA was isolated from anticoagulated blood with DNAzol reagent (Molecular Research Center, Cincinnati, Ohio). Genotyping was performed by using a previously described low-attenuation DNA microarray based on allele-specific probes with minor modifications. ${ }^{30}$

\section{Image Acquisition and Creation of Lesion Masks}

Different image-acquisition protocols were used for the various clinical studies from which our patients were sampled. We used 2D conventional or fast spin-echo proton-attenuation and T2-weighted images, acquired by using either 1T Magnetom Impact (77.4\% of patients) or 1.5T Magnetom Vision (Siemens, Erlangen, Germany) $(22.6 \%)$ scanners by using standard head coils, with section thicknesses of 3-5 mm, in-plane resolution of $1 \times 1 \mathrm{~mm}^{2}$, and a maximum intersection gap of $0.5 \mathrm{~mm}$. On these images, lesions were identified by an expert reader and then were outlined by using home-developed semiautomated seed-growing software (Show Images) ${ }^{31}$ based on a local thresholding technique, thus creating binary lesion masks. T2weighted images were created from each patient's T2-weighted image by using Brain Extraction Tool (FSL). ${ }^{32}$ We created a common-space T2 template with $2 \times 2 \times 2 \mathrm{~mm}$ voxels by linearly registering all T2 brain images (allowing 12 degrees of freedom) to the International Consortium for Brain Mapping(Montreal Neurological Institute152) brain image, ${ }^{33}$ by using the registration tool FLIRT (part of FSL, FMRIB Analysis Group, University of Oxford; http://www.fmrib.ox. ac.uk), ${ }^{34}$ followed by averaging and smoothing by using a 4-mm full width at half maximum Gaussian kernel. To bring all lesion masks to this common space template, we then registered all individual T2 brain images to the common space $\mathrm{T} 2$ brain template (again by using FLIRT with 12 degrees of freedom), and we then applied the registration matrices thus obtained to the corresponding binary lesion masks. Nearest-neighbor interpolation was used to generate individual lesion maps that showed lesion presence or absence for each voxel. Registration quality was ensured by visual inspection. To investigate the effect of the global whole-brain lesion load, we calculated total brain lesion volume. To account for head size differences, we calculated lesion volumes in common space after registration (therefore representing relative lesion volumes).

\section{Statistical Analysis}

Voxel- and clusterwise statistical inference was accomplished by using the nonparametric Randomise method as implemented in FSL 4.0 (FMRIB Analysis Group), by using a GLM design to model each voxel 


\begin{tabular}{|c|c|c|c|c|}
\hline & $\begin{array}{c}\text { Total MS Group: } \\
\quad(N=208)\end{array}$ & $\begin{array}{c}\text { RRMS } \\
(n=126)\end{array}$ & $\begin{array}{c}\text { SPMS } \\
(n=42)\end{array}$ & $\begin{array}{c}\text { PPMS } \\
(n=40)\end{array}$ \\
\hline No. males (\%) & $77(37 \%)$ & $37(29.4 \%)$ & $25(59.5 \%)$ & $15(37.5 \%)$ \\
\hline Age (yr) (mean) ( $\pm S D)$ & $41.1(11.1)$ & $35.6(8.6)$ & $47.8(7.7)$ & $51.6(9.7)$ \\
\hline Disease duration (yr) (median) (IQR) & $6.3(10.1)$ & $3.9(7.8)$ & $11.8(12.2)$ & $8.3(9.5)$ \\
\hline Lesion volume ${ }^{\text {a }}(\mathrm{mL})$ (median) (IOR) & $12.4(35.5)$ & $10.3(22.9)$ & $38.6(102.4)$ & $13.5(40.0)$ \\
\hline EDSS score (median) (IQR) & $3.5(2.9)$ & $2.0(2.0)$ & $6.0(2.5)$ & $4.5(2.0)$ \\
\hline
\end{tabular}

a Lesion volumes were calculated in common space (therefore representing relative lesion volumes).

independently from the other voxels (5000 permutations; clusterforming threshold pseudo- $\mathrm{t}=2$, corresponding to voxelwise P-threshold $=0.01) .{ }^{34,35}$ For each SNP, we made 3 comparisons to test the association of genotypes with voxelwise lesion probability, always comparing 1 genotype with the combination of both other genotypes (ie, the comparisons made were the following: homozygote frequent allele versus heterozygote and other homozygote; homozygote rare allele versus heterozygote and other homozygote; and heterozygote versus both homozygotes). The lesion masks of the patients carrying a certain genotype were put together and compared with the lesion masks of patients who did not carry this genotype. For each comparison, we separately tested for both increased and decreased lesion probability associated with that genotype.

Although SNP markers with low genotype frequencies may be of importance in complex trait disorders like MS, such markers can result in unreliable observations in voxelwise analyses. Therefore, homozygote genotypes with frequencies below $5 \%$ in our cohort were combined with the heterozygote genotype and compared with the other homozygote genotype.

Because total brain lesion volume has been found to influence lesion distribution, ${ }^{7}$ we took the following 2 steps: First, for genotypes showing significant clusters in the LPM analyses, these LPM analyses were repeated while statistically controlling for total brain lesion volume. Second, we investigated the effect of genotype on the total brain lesion volume at a patient level by directly comparing the total brain lesion volume among genotypes, while statistically controlling for disease duration (without taking anatomic distribution into account).

Finally, for the LPM and total brain lesion volume analyses, we tested whether correction for disease duration affected the results by using GLM (after log-transformation of the total lesion volume to obtain a normal distribution).

\section{Results}

\section{Patient Characteristics}

Our cohort of 208 patients with MS was representative of the general MS population regarding patient characteristics ( Table 1), with a slightly high proportion of patients with primary progressive MS (19.2\%). These patients had a quasisymmetric brain lesion distribution typical for MS (Fig 1), with the highest lesion frequencies in the periventricular regions.

\section{Correlations between Genotype and Spatial Lesion Distribution}

Of the 69 SNPs analyzed, 11 genotypes of 10 SNPs showed significant clusters of either increased or decreased lesion probability, predominantly in periventricular clusters, for 1 of the genotypes (Figs 2 and 3). All clusters were located periventricularly, abutting the frontal or occipital horn of the lateral ventricles, often in an asymmetric configuration.
An increased lesion probability, in different periventricular regions, was observed for the heterozygous genotype of 3 SNPs: rs2227139 (within the MHC class II region), rs2076530 (within BTNL2), and rs876493 (within PNMT). Furthermore, there were 2 SNPs in which the homozygous major allele was associated with a higher lesion probability — that is, rs2107538 within CCL5 and rs9808753 within IFNGR2 (Fig 2).

For 6 SNPs, a genotype was associated with a decreased lesion probability (for details on cluster location and genotypes see Fig 3). These 6 SNPs were located within the following genes: BTNL2 (MHC class II-associated), CRYAB, NDUFS7, UCP2, and 2 SNPs within FAS.

When total brain lesion volume was included in the statistical model, only rs2227139 (within the MHC class II region) retained a significant cluster in which the CT genotype was associated with an increased local lesion probability compared with the other 2 genotypes (Fig $2 F$ ).

This result of rs2227139 also remained after correction for disease duration.

To corroborate our findings, we calculated the voxelwise average lesion frequencies for each of the 3 genotypes of the SNP rs2227139, the only SNP that remained significant after controlling for total brain lesion volume. In the voxel with the maximum pseudo-t value observed in the significant cluster (voxelwise pseudo- $\mathrm{t}=4.1$ ), a lesion was present in $29 \%$ ( $n=$ 26/89) of the patients with the heterozygote genotype CT, compared with $16 \%(n=12 / 77)$ of the patients with the CC genotype and $2 \%(n=1 / 42)$ of the patients with the TT genotype.

There were no genotypes of any SNP associated simultaneously with both a decreased lesion probability in 1 location and an increased lesion probability in another, indicating consistency of the observed relations across the brain.

\section{Influence of Genotype on Total Brain Lesion Volume}

We assessed whether genotype was directly related to total brain lesion volume for the SNPs with significant results in the clusterwise analysis. Of the 11 genotypes associated with an increased or decreased lesion probability at the described locations, 6 genotypes were significantly associated with total lesion volume in the brain (Table 2). When we controlled these results for disease duration, 3 SNPs were significantly associated with total lesion volume. The results were consistent with the LPM results - that is, genotypes associated with a higher total brain lesion volume were also associated with an increased local lesion probability and also a decreased local lesion probability. 


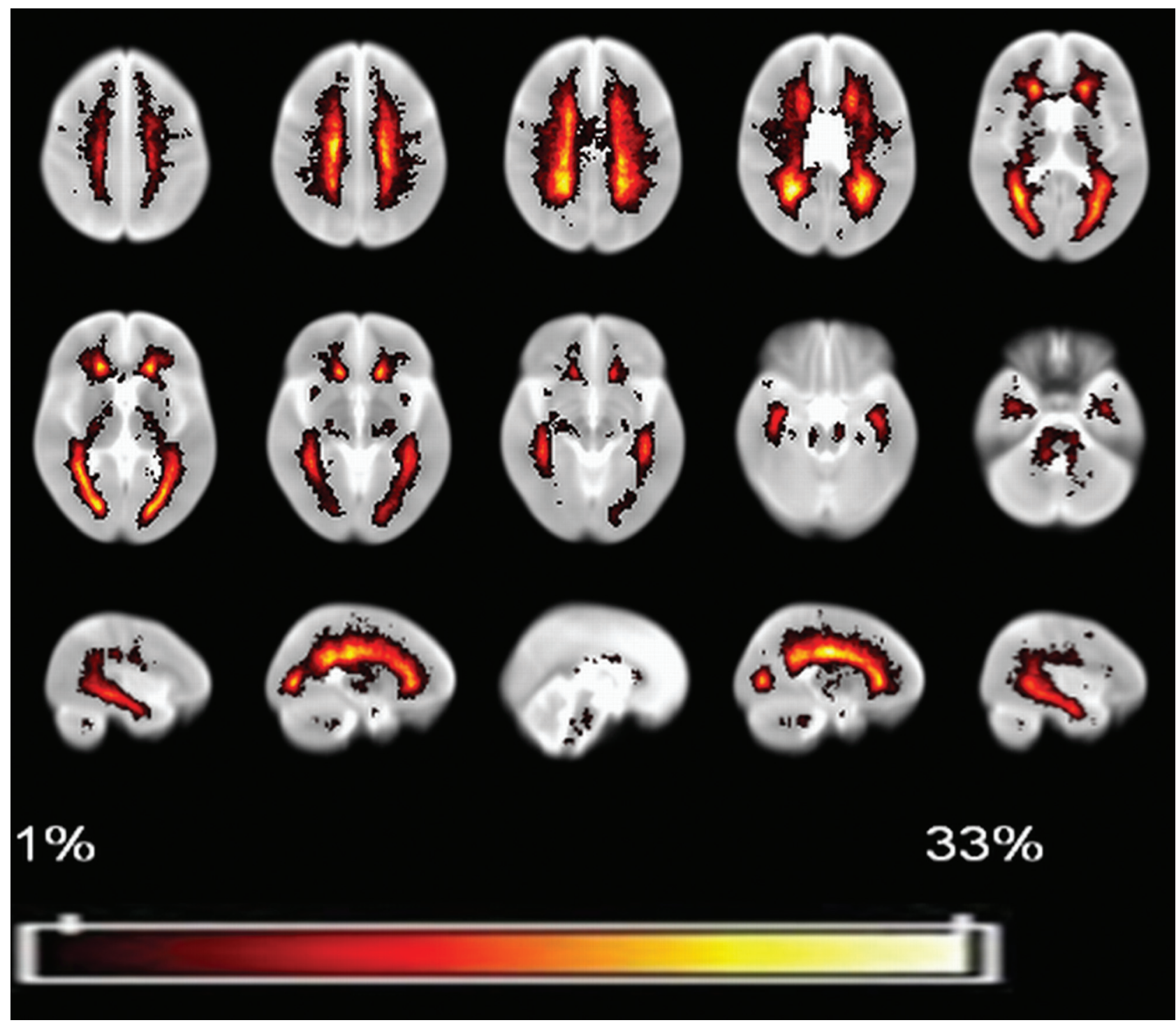

Fig 1. Lesion-frequency map for our group of 208 patients with MS, indicating, for every voxel, the lesion frequency throughout our patient sample, showing a range from $1 \%$ ( $n=2$ patients having a lesion in that voxel) through the maximum of $33 \%(n=69)$.

\section{Discussion}

On the basis of the hypothesis that genetic background may determine spatial lesion patterns, this exploratory study combined lesion probability mapping with genetic data of 69 SNPs in 208 patients with MS. To our knowledge, this is the first study in a large cohort of patients with MS that tests the role of several genes on lesion distribution within the brain. Five SNPs showed a locally increased lesion probability in certain brain regions for 1 genotype, while 6 SNPs showed a decreased lesion probability for 1 of the genotypes. The most robust finding was the increased probability of having a lesion in the left periventricular region next to the frontal and, to a lesser extent, the occipital horn of the ventricles, for the heterozygous genotype of rs2227139 (Fig $2 E,-F$ ). This was the only SNP for which a significant cluster remained after applying a correction for total brain lesion volume. Rs2227139 is located on chromosome 6 within the highly polymorphic MHC class II region, which is involved in self-versus-nonself immune recognition. ${ }^{36}$ These genes have consistently been shown to have a major effect on susceptibility to MS and other autoim- mune diseases ${ }^{36}$ and may be involved in disease severity as well. ${ }^{15,37}$

No data are available on the effect of this SNP on regionspecific differences in the brain. However, previously, multiple HLA alleles showed effects on several MR imaging severity markers, such as T2 lesion volume, T1 hypointense lesion volume, and atrophy measures, ${ }^{38,39}$ suggesting a role for several $H L A$ alleles in the MS phenotype. These findings highlight the importance of $H L A$ genes on MR imaging severity markers. Future studies on the genetic influence on lesion location should preferably include high-resolution HLA typing, enabling comparison of the effect of different HLA alleles on lesion distribution (taking possible interaction between genes into account) and detecting the true causative alleles.

As can be seen from Figs 2 and 3, the SNPs showed altered lesion probability in predominantly 4 regions: the bilateral periventricular white matter next to the frontal and occipital tip of the horns of the lateral ventricles. The periventricular clusters are distributed in an asymmetric manner. We have no explanation for these asymmetries, which persisted after post 


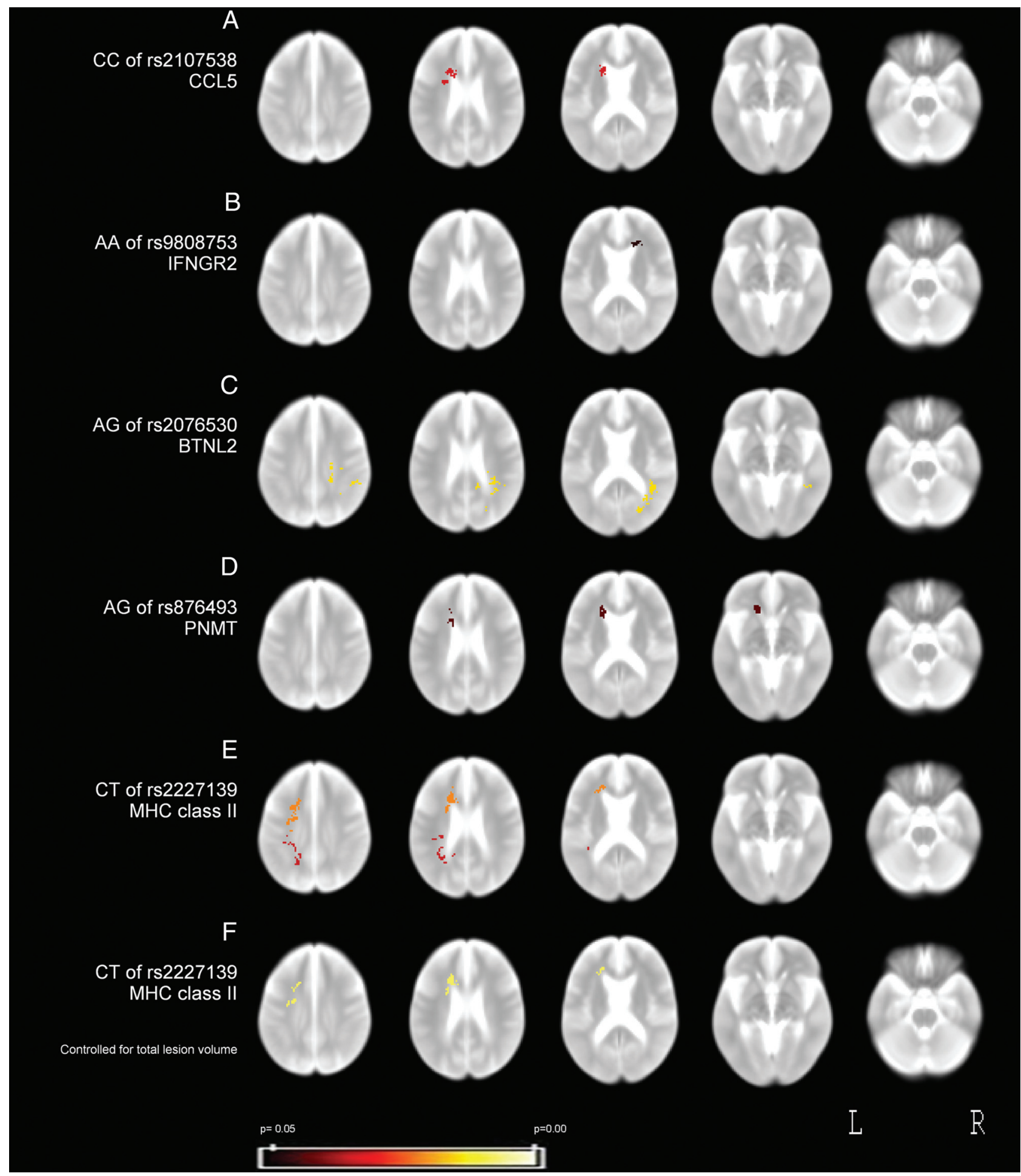

Fig 2. Association between increased lesion probability and genotype. Each row shows results for the comparison of 1 genotype with the other 2 . $A$, CC genotype of rs2107538 within the CCL5 gene. B, AA genotype of rs9808753 within IFNGR2 gene. C, AG genotype of rs2076530 within BTNL2 (MHC class II associated). D, AG genotype of rs876493 (within PNMT). E CT genotype of rs2227139 (within the MHC class II region). F, CT genotype of rs2227139 (within the MHC class II region), when statistically controlled for total T2 brain lesion volume. Images show several axial sections of the T2-weighted template (the same sections in each case), with color overlay (indicating $P$ values) of the clusters in which local lesion probability was significantly increased.

hoc repeated analyses with a lower significance threshold. A priori, a genetic influence on this asymmetric distribution is theoretically unlikely. Another study also noted an asymmetric distribution of MS brain lesions at the individual patient level, possibly reflecting a different lineage pattern. ${ }^{9}$ Although our number of patients is relatively high, bias due to, for ex- ample, the high number of patients with progressive MS is possible and cannot be excluded.

Rs2076530 within the BTNL2 gene was the only SNP showing significant effects for more than 1 genotype. The AG genotype was associated with an increased lesion probability in the periventricular white matter adjacent to the right occipital horn, 


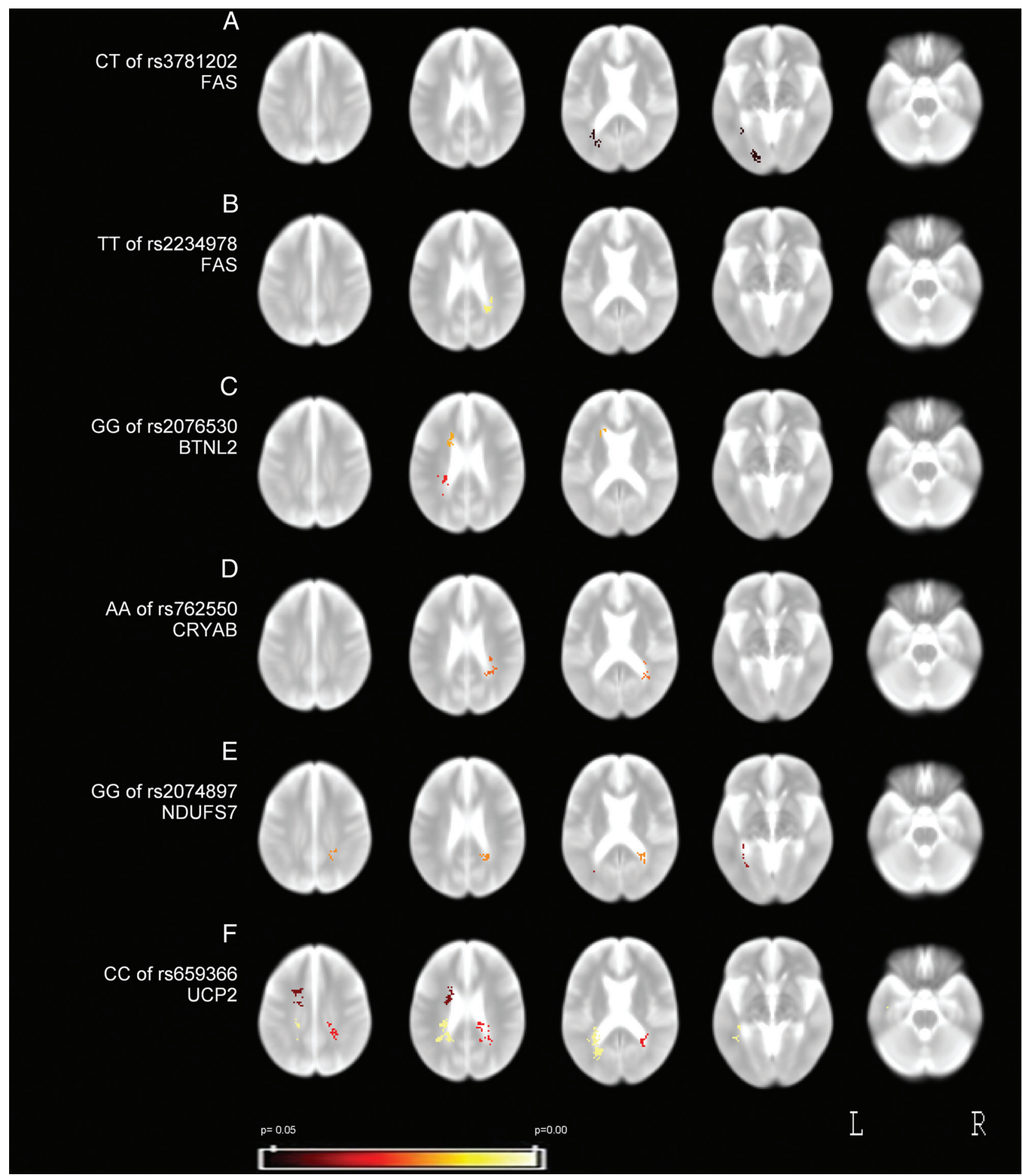

Fig 3. Association between decreased lesion probability and genotype. Each row shows the results for the comparison of 1 genotype with the other 2 . $A$, CT genotype of rs3781202 (within FAS). B, TT genotype of rs2234978 (within FAS). C, GG genotype of rs2076530 within BTNL2 (MHC class II associated). D, AA genotype of rs762550 within CRYAB. E, GG genotype of rs2074897 within NDUFS7. F, CC genotype of rs659366 within UCP2. Images show several axial sections of the T2-weighted template (the same sections in each case), with color overlay of the clusters in which local lesion probability was significantly decreased.

while the GG genotype was associated with a decreased lesion probability in the periventricular white matter adjacent to the left occipital and frontal horns. Both effects were nonconflicting and disappeared after correction for total brain lesion volume.

The relation between $H L A-D R B 1^{*} 1501$ (the MHC class II allele strongly associated with susceptibility to MS) ${ }^{12,40,41}$ and spatial lesion distribution within the brain was studied in 1 previous report ${ }^{23}$ demonstrating no significant influence of the allele. Our results (by using rs3135388, a SNP in the MHC region predicting the $H L A-D R B 1^{*} 1501$ haplotype with very high sensitivity) ${ }^{28}$ confirmed, in a larger sample, the negative results of this previous study of Sepulcre et al. ${ }^{23}$ In a subset of 


\begin{tabular}{|c|c|c|c|c|c|}
\hline \multirow[b]{2}{*}{ Gene } & \multirow[b]{2}{*}{ rs No. } & \multicolumn{2}{|c|}{$\begin{array}{l}\text { Mean Whole Brain T2 Lesion } \\
\text { Volumes in } \mathrm{mL} \text { (SD) }\end{array}$} & \multicolumn{2}{|c|}{$P$ Value of Differences in Total Lesion Volume } \\
\hline & & $\begin{array}{l}\text { Genotype of } \\
\text { Interest }^{\mathrm{a}}\end{array}$ & Other Genotypes & $\begin{array}{l}\text { Between Genotypes } \\
\text { (Mann-Whitney } U \text { test) }\end{array}$ & $\begin{array}{l}\text { Corrected for Disease } \\
\text { Duration (using GLM) }\end{array}$ \\
\hline \multicolumn{6}{|c|}{ Increased local volume in LPM analyses } \\
\hline BTNL2 & rs2076530 b & AG $32.0(32.9)$ & AA and GG 23.8 (27.4) & $0.04^{\mathrm{c}}$ & $0.05^{\mathrm{c}}$ \\
\hline CCL5 & rs2107538 & CC $28.2(29.0)$ & CT and $\mathrm{TT}^{\mathrm{d}} 20.9(28.1)$ & $0.02^{\mathrm{c}}$ & 0.07 \\
\hline IFNGR2 & rs9808753 & AA $28.5(30.5)$ & $\mathrm{AG}$ and $\mathrm{GG}^{\mathrm{d}} 18.3(22.6)$ & $0.04^{\mathrm{c}}$ & 0.29 \\
\hline PNMT & rs876493 & AG $28.1(29.0)$ & $\mathrm{AA}$ and GG $23.2(28.6)$ & 0.15 & 0.13 \\
\hline HLA & rs2227139 & СT $28.0(29.9)$ & CC and TT $23.9(28.0)$ & 0.35 & 0.25 \\
\hline \multicolumn{6}{|c|}{ Decreased local lesion probability in LPM analyses } \\
\hline BTNL2 & rs $2076530^{c}$ & GG $18.7(22.7)$ & $A A$ and $A G 26.6(29.5)$ & 0.13 & $0.04^{\mathrm{C}}$ \\
\hline CRYAB & rs762550 & AA $19.8(21.0)$ & $A G$ and $G G 27.0(30.2)$ & 0.28 & 0.30 \\
\hline FAS & rs2234978 & TT 12.5 (14.7) & CC and CT 27.1 (29.7) & $0.02^{\mathrm{c}}$ & 0.06 \\
\hline FAS & rs3781202 & CT 23.1 (28.5) & CC and TT $28.4(29.1)$ & 0.18 & 0.37 \\
\hline NDUFST & rs2074897 & GG 18.4 (23.7) & $A A$ and $A G 28.2(30.1)$ & $<.01^{\mathrm{C}}$ & 0.08 \\
\hline UCP2 & rs659366 & CC 18.6 (23.1) & CT and TT 29.8 (31.1) & $<.01^{\mathrm{c}}$ & $<.01^{\mathrm{C}}$ \\
\hline
\end{tabular}

a Genotypes that are significantly associated with altered local lesion probability in LPM analyses.

${ }^{b}$ Different genotypes of this same SNP are involved in decreased and increased lesion probability.

c $P$ values of significant associations to total T2 lesion volume.

d Because of the frequency of $<5 \%$ of homozygote genotype, these genotypes (TT for CCL5 and GG for IFNGR2) were only analyzed when combined with heterozygote genotypes.

the current patient group, we previously found the HLA$D R B 1^{*} 1501$ allele to be related to increased lesion loads in the spinal cord but not the brain. In the present study on a larger patient group encompassing the former, we confirmed that the HLA-DRB ${ }^{*} 1501$ allele was not related to the total volume of brain lesions, ${ }^{22}$ and most important, we added the new finding that it is also unrelated to their anatomic distribution across the brain.

Limitations of this study include the relatively low field strength of the MR imaging scanners we used, which, in general, leads to lower sensitivity to lesions than current state-ofthe-art MR imaging scanners operating at 3T or above. ${ }^{42}$

Furthermore, the linear registration method used to warp the lesion masks of individual patients to the template, by definition, cannot correct for the variability in ventricular and sulcal sizes that is present in patients with MS as a result of differences in brain atrophy. Therefore, the matching between individual patients' brains will be imperfect; this discrepancy leads to less accurate overlay of corresponding anatomic areas and may explain, to some extent, why we only found clusters in the periventricular region and not in areas with a lower lesion frequency. Despite these limitations, we deliberately chose a linear registration method (FLIRT) because of the difficulties associated with nonlinear registration methods. In this study, we were limited in the sequences available (dualecho images only); this limitation poses problems for nonlinear registration regarding the distinction between periventricular lesions and ventricular CSF because of their similar signal intensities. Second, nonlinear registration could result in displacement of lesions inside the brain, thereby affecting the very aspect of the disease that we intended to study (namely lesion distribution).

The third limitation is the absence of gray matter lesions in our study, because these lesions go mostly undetected when using standard MR imaging techniques. ${ }^{43}$ From postmortem studies we know that gray matter lesions are extensively present in patients with MS, ${ }^{43}$ and it can be expected that a different genetic background may influence the lesion distribution between the white and gray matter compartments, as well as the anatomic distribution within the white and gray matter.

Total brain lesion volume seems to be an important covariate in LPM analyses. ${ }^{7}$ Apart from the left frontal horn cluster in rs2227139, no clusters retained significance when the model was controlled for lesion volume. Thus, the influence of genetic background on spatial lesion distribution, as assessed in the current study, may partly act through total brain lesion volume and come to expression in voxelwise analyses, especially in regions with relatively high frequencies of MS lesions, where differences among groups can become statistically significant (ie, the periventricular white matter). Therefore, in future studies, when comparing groups, a correction for total brain lesion volume should be considered.

The genes investigated in this study are putatively associated with susceptibility or disease severity in MS, and we hypothesized that the variation in anatomic distribution of brain lesions among patients with MS may be partly genetic in nature. Our results suggest that such a genetic influence may indeed be present. Independent studies should now be conducted to confirm these findings, including studies using alternative methodology in addition to LPM, for example, by using predefined regions of interest defined on the basis of LPM results to increase sensitivity in detecting differences. For the genes with significant results, there are no obvious relationships between their functions and spatial MS lesion distribution. However, several of the SNPs that were significantly associated with lesion distribution were also significantly associated with the total lesion volume in the brain. The previously reported effect of these genes on the overall lesion burden (T2 lesion volume, T1 hypointense lesion volume, or atrophy ${ }^{18,38,44,45}$ ), acting through their functions in different processes such as mitochondrial energy metabolism and inflammation, is likely to generate differences in lesion distribution among the genotypes.

Our finding that statistically controlling for total brain lesion volume removes the observed effects on lesion distribution suggests that these genes possibly exert an effect mainly on 
the overall lesion burden. However, to fully address this issue, a more focused approach should be applied, by using both an LPM approach and predefined areas of interest (hereby increasing the statistical power) and by selecting more homogeneous patients to limit the effect of confounding factors. In this context, a longitudinal study on the spatiotemporal development of new lesions (including T1 hypointense lesions and T1 gadolinium-enhancing lesions) would be valuable. Eventually, the insights gained from this approach may yield ways of predicting, from patients' genotypes, whether they are likely to develop lesions in clinically eloquent areas.

Recently, pathway analysis in relation to susceptibility to MS revealed, not surprisingly, immune-related pathways. ${ }^{46}$ However, the same study also detected significant neural pathways, implicating a primary neural dysfunction in susceptibility to MS. This pathway-analysis approach may provide a basis for patient stratification (for instance, patients carrying a predominantly inflammatory profile versus patients carrying a more neurodegenerative profile), and it would be valuable to characterize these patient groups in terms of their lesion distribution. Previously, some of the SNPs selected for this study were found related, in relatively small studies, to a more neurodegenerative profile (CCR5 and CRYAB), ${ }^{18,45}$ while others have found several HLA alleles associated with a more inflammatory profile. ${ }^{37,38}$ Such profile differences may be able to explain the previously described differences in lesion distribution between $\mathrm{T} 1$ gadolinium-enhancing lesions (acute inflammatory activity) and $\mathrm{T} 1$ persistent hypointense lesions (neurodegenerative activity). ${ }^{9}$

\section{Conclusions}

This exploratory study revealed an association between heterozygosity on rs2227139 in the MHC class II region and preferential periventricular lesion formation in MS. Other SNPs also showed associations between genotype and spatial lesion distribution, which are worth studying in future investigations by using a more focused approach and considering genetic pathways in addition to single genes. In these future studies, the potential role of lesion volume influencing the relation between genotype and lesion distribution should be explored further, as well as the distribution of gray matter lesions, contrast-enhancing lesions, and persistent T1-hypointense lesions.

\section{Acknowledgments}

We thank all patients for participating.

\section{References}

1. Lucchinetti C, Bruck W, Parisi J, et al. Heterogeneity of multiple sclerosis lesions: implications for the pathogenesis of demyelination. Ann Neurol. 2000;47:707-17

2. Hooper-van Veen T, Berkhof J, Polman CH, et al. Analysing the effect of candidate genes on complex traits: an application in multiple sclerosis. Immunogenetics 2006;58:347-54. Epub 2006 Apr 13

3. Narayanan S, Fu L, Pioro E, et al. Imaging of axonal damage in multiple sclerosis: spatial distribution of magnetic resonance imaging lesions. Ann Neurol 1997;41:385-91

4. Barkhof F, Filippi M, Miller DH, et al. Comparison of MRI criteria at first presentation to predict conversion to clinically definite multiple sclerosis. Brain 1997;120(pt 11):2059-69

5. van Walderveen MA, Barkhof F, Tas MW, et al. Patterns of brain magnetic resonance abnormalities on $\mathrm{T} 2$-weighted spin echo images in clinical subgroups of multiple sclerosis: a large cross-sectional study. Eur Neurol 1998;40:91-98

6. Di Perri C, Battaglini M, Stromillo ML, et al. Voxel-based assessment of differences in damage and distribution of white matter lesions between patients with primary progressive and relapsing-remitting multiple sclerosis. Arch Neurol 2008;65:236-43

7. Vellinga MM, Geurts JJ, Rostrup E, et al. Clinical correlations of brain lesion distribution in multiple sclerosis. J Magn Reson Imaging 2009;29:768-73

8. Lee MA, Smith S, Palace J, et al. Spatial mapping of T2 and gadolinium-enhancing T1 lesion volumes in multiple sclerosis: evidence for distinct mechanisms of lesion genesis? Brain 1999;122(pt 7):1261-70

9. Koziol JA, Wagner S, Sobel DF, et al. Asymmetries in the spatial distributions of enhancing lesions and black holes in relapsing-remitting MS. J Clin Neurosci 2005;12:895-901

10. Dyment DA, Ebers GC, Sadovnick AD. Genetics of multiple sclerosis. Lancet Neurol 2004;3:104-10

11. Hafler DA, Compston A, Sawcer S, et al. Risk alleles for multiple sclerosis identified by a genomewide study. N Engl J Med 2007;357:851-62

12. Sawcer S, Ban M, Maranian M, et al. A high-density screen for linkage in multiple sclerosis. Am J Hum Genet 2005;77:454-67

13. Baranzini SE, Wang J, Gibson RA, et al. Genome-wide association analysis of susceptibility and clinical phenotype in multiple sclerosis. Hum Mol Genet 2009;15;18:767-78. Epub 2008 Nov 14

14. Barcellos LF, Oksenberg JR, Green AJ, et al. Genetic basis for clinical expression in multiple sclerosis. Brain 2002;125:150-58

15. DeLuca GC, Ramagopalan SV, Herrera BM, et al. An extremes of outcome strategy provides evidence that multiple sclerosis severity is determined by alleles at the HLA-DRB1 locus. Proc Natl Acad Sci U S A 2007;104:20896-901. Epub 2007 Dec 17.

16. Hoffmann S, Cepok S, Grummel V, et al. HLA-DRB ${ }^{\star} 0401$ and HLADRB $1^{\star} 0408$ are strongly associated with the development of antibodies against interferon-beta therapy in multiple sclerosis. Am J Hum Genet 2008;83:219-27

17. Kantarci $\mathrm{OH}$, de Andrade M, Weinshenker BG. Identifying disease modifying genes in multiple sclerosis. J Neuroimmunol 2002;123:144-59

18. van Veen T, van Winsen L, Crusius JB, et al. [Alpha]B-crystallin genotype has impact on the multiple sclerosis phenotype. Neurology 2003;61:1245-49

19. Deen S, Bacchetti P, High A, et al. Predictors of the location of multiple sclerosis relapse. J Neurol Neurosurg Psychiatry 2008;79:1190-93

20. Mowry EM, Deen S, Malikova I, et al. The onset location of multiple sclerosis predicts the location of subsequent relapses. J Neurol Neurosurg Psychiatry 2009;80:400-03

21. Butterfield RJ, Blankenhorn EP, Roper RJ, et al. Identification of genetic loci controlling the characteristics and severity of brain and spinal cord lesions in experimental allergic encephalomyelitis. Am J Pathol 2000;157:637-45

22. Sombekke MH, Lukas C, Crusius JB, et al. HLA-DRB1*1501 and spinal cord magnetic resonance imaging lesions in multiple sclerosis. Arch Neurol 2009;66:1531-36

23. Sepulcre J, Masdeu JC, Palacios R, et al. HLA-DR2 and white matter lesion distribution in MS. J Neuroimaging 2008;18:328-31

24. Poser CM, Paty DW, Scheinberg L, et al. New diagnostic criteria for multiple sclerosis: guidelines for research protocols. Ann Neurol 1983;13:227-31

25. McDonald WI, Compston A, Edan G, et al. Recommended diagnostic criteria for multiple sclerosis: guidelines from the international panel on the diagnosis of multiple sclerosis. Ann Neurol 2001;50:121-27

26. Polman $\mathrm{CH}$, Reingold SC, Edan G, et al. Diagnostic criteria for multiple sclerosis: 2005 revisions to the "McDonald Criteria." Ann Neurol 2005;58:840-46

27. Kurtzke JF. Rating neurologic impairment in multiple sclerosis: an expanded disability status scale (EDSS). Neurology 1983;33:1444-52

28. de Bakker PI, McVean G, Sabeti PC, et al. A high-resolution HLA and SNP haplotype map for disease association studies in the extended human MHC. Nat Genet 2006;38:1166-72

29. Godde R, Rohde K, Becker C, et al. Association of the HLA region with multiple sclerosis as confirmed by a genome screen using $>10,000$ SNPs on DNA chips. J Mol Med 2005;83:486-94

30. Tejedor D, Castillo S, Mozas P, et al. Reliable low-density DNA array based on allele-specific probes for detection of 118 mutations causing familial hypercholesterolemia. Clin Chem 2005;51:1137-44

31. van Walderveen MA, Barkhof F, Hommes OR, et al. Correlating MRI and clinical disease activity in Multiple Sclerosis: relevance of hypointense lesions on shortTR/short-TE (T1-weighted) spin echo images. Neurology 1995;45:1684-90.

32. Smith SM. Fast robust automated brain extraction. Hum Brain Mapp 2002;17:143-55

33. Mazziotta JC, Toga AW, Evans A, et al. A probabilistic atlas of the human brain: theory and rationale for its development-The International Consortium for Brain Mapping (ICBM). Neuroimage 1995;2:89-101

34. Smith SM, Jenkinson M, Woolrich MW, et al. Advances in functional and structural MR image analysis and implementation as FSL. Neuroimage 2004; 2(suppl 1):S208-19 
35. Nichols TE, Holmes AP. Nonparametric permutation tests for functional neuroimaging: a primer with examples. Hum Brain Mapp 2002;15:1-25

36. Fernando MM, Stevens CR, Walsh EC, et al. Defining the role of the MHC in autoimmunity: a review and pooled analysis. PLoS Genet 2008;4:e1000024

37. Zivadinov R, Uxa L, Zacchi T, et al. HLA genotypes and disease severity assessed by magnetic resonance imaging findings in patients with multiple sclerosis. J Neurol 2003;250:1099-106

38. Zivadinov R, Uxa L, Bratina A, et al. HLA-DRB1*1501, -DQB1*0301, $-D Q B 1{ }^{\star} 0302,-D Q B 1{ }^{*} 0602$, and $-D Q B 1{ }^{*} 0603$ alleles are associated with more severe disease outcome on MRI in patients with multiple sclerosis. Int Rev Neurobiol 2007;79:521-35

39. Healy BC, Liguori M, Tran D, et al. HLA B ${ }^{\star} \mathbf{4 4}$ : protective effects in MS susceptibility and MRI outcome measures. Neurology 2010;75:634-40

40. Barcellos LF, Oksenberg JR, Begovich AB, et al. HLA-DR2 dose effect on susceptibility to multiple sclerosis and influence on disease course. Am J Hum Genet 2003;72:710-16

41. Lincoln MR, Montpetit A, Cader MZ, et al. A predominant role for the HLA class II region in the association of the MHC region with multiple sclerosis. Nat Genet 2005;37:1108-12

42. Wattjes MP, Lutterbey GG, Harzheim M, et al. Higher sensitivity in the detection of inflammatory brain lesions in patients with clinically isolated syndromes suggestive of multiple sclerosis using high field MRI: an intraindividual comparison of 1.5 T with 3.0 T. Eur Radiol 2006;16:2067-73

43. Geurts JJ, Barkhof F. Grey matter pathology in multiple sclerosis. Lancet Neurol 2008;7:841-51

44. Witte ME, Bo L, Rodenburg RJ, et al. Enhanced number and activity of mitochondria in multiple sclerosis lesions. J Pathol. 2009;219:193-204

45. van Veen T, Nielsen J, Berkhof J, et al. CCL5 and CCR5 genotypes modify clinical, radiological and pathological features of multiple sclerosis. J Neuroimmunol 2007;190:157-64

46. Baranzini SE, Galwey NW, Wang J, et al. Pathway and network-based analysis of genome-wide association studies in multiple sclerosis. Hum Mol Genet 2009;18:2078-90 